\title{
The statistical effects of measuring myocytes with different image zoom rates
}

\author{
This article was published in the following Dove Press journal: \\ Open Access Medical Statistics \\ 25 May $201 \mathrm{I}$ \\ Number of times this article has been viewed
}

\section{Chengxin $\mathrm{Li}$ \\ Maiying Kong \\ Dongfeng Wu}

Department of Bioinformatics and Biostatistics, School of Public Health and Informatics Science, University of Louisville, Louisville, KY, USA
Correspondence: Maiying Kong Department of Bioinformatics and Biostatistics, School of Public Health and Informatics Science, 485 E Gray Street, Louisville, KY 40202, USA

Tel +l 5028523988

$\mathrm{Fax}+\mathrm{I} 5028523294$

Email maiying.kong@louisville.edu
Abstract: Quantitative image measurements are widely used in both basic and clinical medical research. The measurements can be taken under different image zoom rates. Usually, increasing the image zoom rate increases the accuracy of the measurement; however, increasing the image zoom rate also increases the measurement cost. We measured 218 rat myocytes (112 myocytes from treated rat heart; 106 from placebo rat heart) for image measurements of cross-sectional areas, under different image zoom rates, $75 \%, 100 \%, 150 \%, 200 \%$, and $300 \%$. The linear mixedeffects model, Student's $t$-test, and $F$ test were used to investigate whether image measurements significantly change over different zoom rates, and to find the optimal zoom rate in terms of maximizing the test statistic while reducing measurement cost. We found that the means of the measured myocyte areas decreased with increased zoom rates for both the treated heart and the placebo heart. The means of measured areas at zoom rate $75 \%$ versus $100 \%$ were not significantly different for both the treated heart and the placebo heart. However, there was a significant decrease when the zoom rate increased from $100 \%$ to $150 \%$ for the treated heart and the placebo heart, and the amount of decrease of the means for the two types of hearts was different. When the zoom rate was increased from $150 \%$ to $200 \%$ and $300 \%$, the mean of the measurements decreased. However, the amount of decrease for both the treated heart and the placebo heart was the same. When image zoom rate increases, measurement precision remains the same. The $t$-test statistics for comparing treated heart and placebo heart remain the same when zoom rate reaches $150 \%$ and above. Therefore, the image zoom rate at $150 \%$ is the optimal choice in terms of maximizing the test statistic and reducing the measurement cost.

Keywords: image zoom rate, myocyte, cross-sectional area measurement, measurement cost, wheat germ agglutinin (WGA) staining, linear mixed-effects model

\section{Introduction}

Quantitative image measurements most often take place in either basic or clinical medical research, such as measurement for myocyte cross-sectional area and for capillary or vessel cross-sectional area. The measurement instruments can usually be adjusted to different magnification rates, which are often called image zoom rates. Larger zoom rate implies that the measured areas are magnified more and the number of experimental units measured per screen becomes less. Generally speaking, increasing the image zoom rate increases the accuracy of the measurement. However, increasing the image zoom rate also increases the measurement cost dramatically in terms of time and effort. Hence, there is a need to obtain an optimal zoom rate in which statistical test power is maximized, measurement cost is reduced, and measurement accuracy and precision are maintained. 
In this study, two rat hearts were used: a stem cell-treated heart (treatment) and a untreated heart (placebo). A total of 218 myocytes were selected, of which 112 myocytes were from the treated heart and 106 were from the placebo heart. For each myocyte, we took the measurements of the myocyte area under different zoom rates. We examined the changes of measurement accuracy and precision with the increasing image zoom rates during image measurement, and derived the optimal image zoom rate for obtaining a high-power test statistic with low measurement cost.

This paper does not cover any treatment effects but focuses only on the measurement effects caused by different image zoom rates. Readers who are interested in the treatment effects may refer to Tang et al. ${ }^{1}$ To simplify the notation, the stem cell-treated heart is hereafter called the treated heart, and the untreated heart is called the placebo heart.

\section{Materials and methods Data collection}

The images were acquired digitally under microscope (20x) in a TIFF format with a default resolution of 72 pixels/inch. The raw data were collected from image measurements of rat myocyte cross-sectional areas, which were determined by planimetry after staining cell membranes with green fluorescein isothiocyanate (FITC)-conjugated wheat germ agglutinin (WGA). The selection of myocytes for measurement was based on a clearly defined sarcolemma board and with associated round nuclei, which were stained with 4',6-diamidino-2-phenylindole (DAPI) (blue). In total, 218 myocytes were selected based on these criteria, of which 112 myocytes were from the treated heart, and 106 were from the placebo heart.

Measurement of the myocyte cross-sectional area was conducted with ImageJ v1.39u software (NIH, Bethesda, $\mathrm{MD})$. ImageJ is an open source package sponsored by the National Institutes of Health (NIH) (http://rsb.info.nih. gov/ij/download.html). Figure 1 provides an illustration of the features of the selected myocytes. The solid blue color shows nuclei stained with DAPI. The green color shows cell membranes stained with FITC-conjugated WGA. The red lines are traced with ImageJ for cell area measurements.

The protocol for measuring the myocyte area is as follows.

1. Open the image with ImageJ $(75 \%$ zoom rate by default).

2. Set up the measurement scale at $3.082 / \mu \mathrm{m}$.

3. For each of the selected 218 myocytes, repeat step 4 and step 5 .

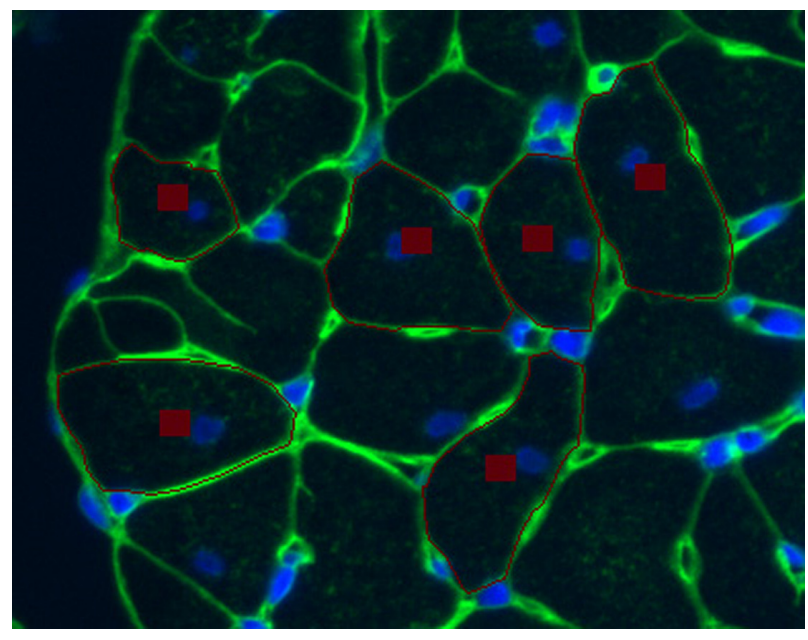

Figure I Illustration of myocyte selection for (see text for explanation of colors).

4. Trace the inner green edge of the myocyte

5. Measure the area

6. For other image zoom rates $100 \%, 150 \%, 200 \%$, and $300 \%$, repeat step 3 to step 5 .

See Table 1 and Appendix II for the illustration of the collected data.

\section{Statistical analysis}

The mean and standard deviation for each type of heart at each zoom rate were calculated as shown in Table 2. We also report the $P$-values for testing the equality of the variances of the two types of hearts at each zoom rate, the pooled standard deviation, the mean difference, and the $t$-test statistic for testing the difference of the measured myocyte areas of the two types of hearts at each zoom rate. The linear mixed-effects model was used to examine whether and how the means of the measured myocyte areas changed over different image zoom rates. ${ }^{2}$ The normality assumption for the linear mixed-effect model is valid based on the Q-Q plots of the measured myocyte areas for each type of heart and at each zoom rate. Student's $t$-tests (paired

Table I Illustration of the collected data

\begin{tabular}{llll}
\hline Area $^{\text {a }}$ & Measurement type $^{\text {b }}$ & Group $^{c}$ & Myocyte $^{\text {d }}$ \\
\hline 259.509 & 75 & Treated & I \\
311.831 & 75 & Treated & 2 \\
255.403 & 75 & Treated & 3 \\
$\ldots$ & $\ldots$ & $\ldots$ & $\ldots$ \\
315.306 & 300 & Placebo & 104 \\
377.946 & 300 & Placebo & 105 \\
379.946 & 300 & Placebo & 106 \\
\hline
\end{tabular}

Notes: ${ }^{\mathrm{a}}$ The area of myocyte in the unit of $\mu \mathrm{m}^{2}$, a dependent variable; ${ }^{\mathrm{b}}$ measurement type, representing different image measurement zoom rates, such as $75 \%, 100 \%$, $150 \%, 200 \%$, and $300 \%$; 'group factor, indicating whether the myocyte came from a treated heart or a placebo heart; 'the identification number for myocyte in each group, taking I-I I 2 for treatment and I-106 for placebo. 


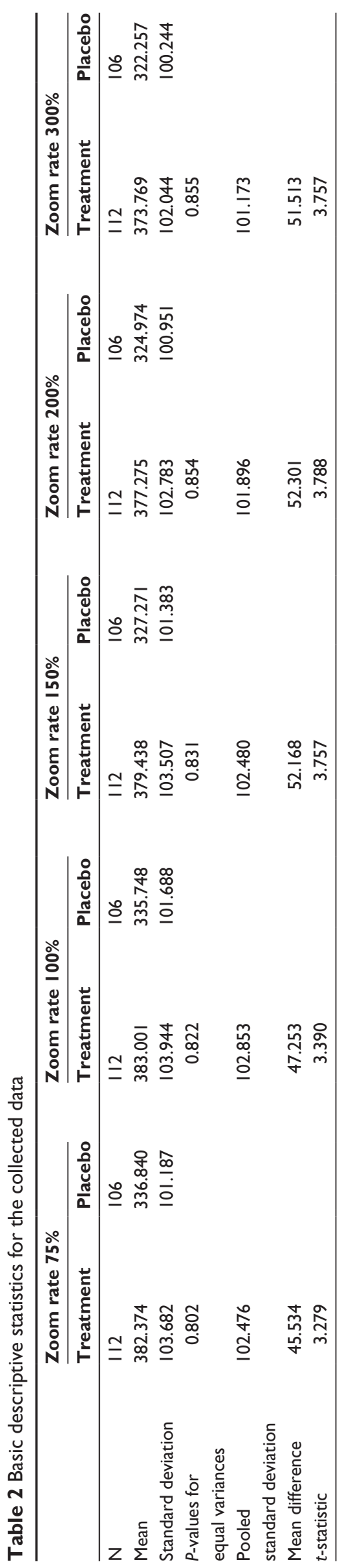

and unpaired) were used for analyses of measurement precision and inference on the optimal zoom rate. ${ }^{3}$ A significant level for $\alpha$ at 0.05 was adopted unless otherwise specified.

To examine whether the different zoom rates significantly change the measurements of the myocyte area, we first plotted the measurements of each myocyte area over different zoom rates $(75 \%, 100 \%, 150 \%, 200 \%$, and $300 \%)$ in Figure 2 as a thin line, where the solid thick line in each panel represents the means of the measured myocyte areas over different zoom rates for either the treated heart or the placebo heart. From Figure 2, it is clear that the between-myocyte variation is much larger than the within-myocyte variation. We used the linear mixed-effects model to examine the contribution of the different zoom rates on the measurements of a myocyte area. Let us denote $y_{i}$ as the measured area for the $i$ th myocyte at different zoom rates, ie, $y_{i}=\left(y_{i 1}, y_{i 2}, y_{i 3}, y_{i 4}, y_{i 5}\right)^{\mathrm{T}}$. The linear mixed-effect model can be specified by the following:

$y_{i j}=\beta_{0}+\beta_{1} I\{$ treatment $\}+\beta_{2} I\{m t 100\}+\beta_{3} I\{m t 150\}$

$+\beta_{4} I\{m t 200\}+\beta_{5} I\{m t 300\}+\beta_{6}\{$ treatment \& mt100\}

$+\beta_{7} I$ treatment \& mt150\} $+\beta_{8}$ Iftreatment \& mt200\}

$+\beta_{9} I\{$ treatment $\& m t 300\}+b_{i}+\varepsilon_{i j}$.

Here, $I\{$ is an indicator function and takes a value of either 0 or 1. For example, if an observation is from the treated heart and is measured at a zoom rate of $100 \%$, then I\{treatment $\}$, $I\{m t 100\}$, and $I$ treatment $\& m t 100\}$ all take values of 1 , and all other indicator functions take values of 0 . With these notations, the expected values for the combination of different hearts and different zoom rates are presented in Table 3 . The $b_{i}$ in model (1) is assumed to be an identically and independently distributed random variable, with a normal distribution with mean 0 and variance $\sigma_{b}^{2}$. The distribution of within-myocyte error $\varepsilon_{i j}$ is usually assumed to be normally distributed with mean 0 and variance $\sigma^{2}$. Generally, $\sigma_{b}^{2}$ describes the between-myocyte variation, and $\sigma^{2}$ describes the within-myocyte variation.

It is generally assumed that the observations for different myocytes are independent, and the within-myocyte observations are correlated. In model (1), the assumption that $b_{i} \sim N\left(0, \sigma_{b}^{2}\right)$ implies that the within-myocyte observations have the same level of correlations, which seems to be a reasonable assumption for this study. The parameters in model (1) can be obtained by using the lme function in the R package nlme, ${ }^{2}$ which can be obtained from http://www.r-project.org.

\section{Results}

For model (1), the fitted results are presented in Table 4. From Table 4: (i) the estimates for $\beta_{2}, \beta_{3}, \beta_{4}$, and $\beta_{5}$ are decreasing, which indicates that the means of the measurements of 

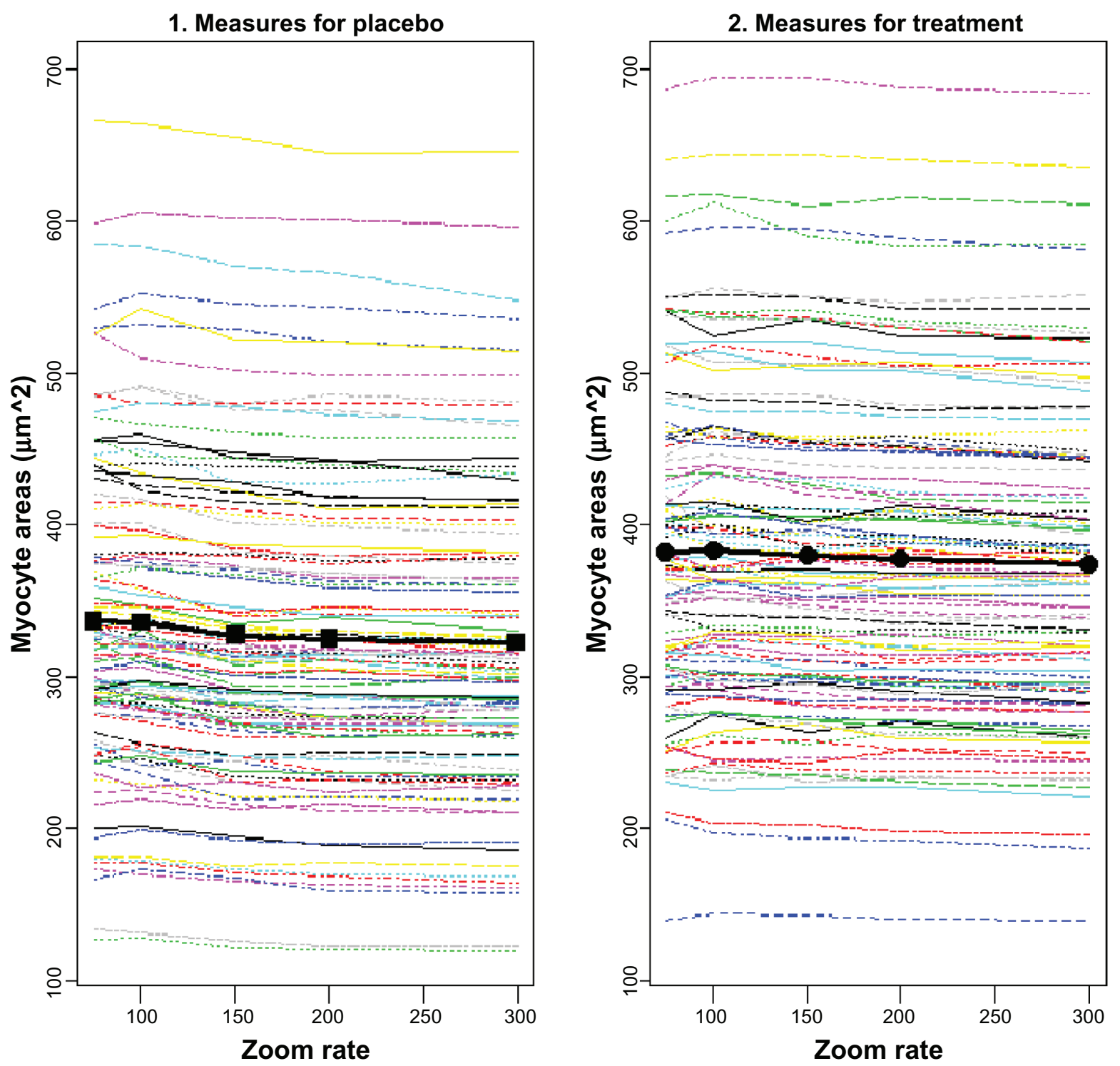

Figure 2 The measured myocyte areas for each myocyte over different zoom rates. The solid thick line in each panel connects the means of the myocyte areas measured at different zoom rates, $75 \%, 100 \%, 150 \%, 200 \%$, and $300 \%$.

myocyte areas for placebo heart are decreasing as the image zoom rate increases; (ii) from $\beta_{2}+\beta_{6}=0.6, \beta_{3}+\beta_{7}=-3.0$, $\beta_{4}+\beta_{8}=-5.1$, and $\beta_{5}+\beta_{9}=-8.6$, we conclude that the means of the measured areas of myocytes of the treated heart are decreasing as the zoom rate increases above $150 \%$; (iii) based on the Wald test statistics, $\beta_{2}$ and $\beta_{6}$ are not significantly different from 0 , which indicate that the means of the measured areas of myocytes at zoom rates $75 \%$ and $100 \%$ are not significantly different; (iv) $\beta_{3}, \beta_{4}$, and $\beta_{5}$ are significantly different from 0 , which indicates that the measured areas of the myocytes from the placebo heart are significantly different across different zoom rates; (v) based on the estimate for $\beta_{2}$, $\beta_{3}, \beta_{4}$, and $\beta_{5}$, the mean change from zoom rate $100 \%$ to $150 \%$ is more dramatic than for any other pairs; (vi) $\beta_{7}, \beta_{8}$, and $\beta_{9}$ are not significantly different, which indicates that the differences of the means for treated heart and placebo heart are not significantly different at the three different zoom rates $150 \%$, $200 \%$, and $300 \%$; and (vii) $\sigma_{b}^{2}=101.6^{2}$ and $\sigma^{2}=4.4^{2}$, which

Table 3 Expected values for different types of hearts at different zoom rates based on model (I)

\begin{tabular}{llllll}
\hline Zoom rate & $\mathbf{7 5 \%}$ & $\mathbf{1 0 0 \%}$ & $\mathbf{1 5 0 \%}$ & $\mathbf{2 0 0 \%}$ & $\mathbf{3 0 0 \%}$ \\
\hline Placebo & $\beta_{0}$ & $\beta_{0}+\beta_{2}$ & $\beta_{0}+\beta_{3}$ & $\beta_{0}+\beta_{4}$ & $\beta_{0}+\beta_{5}$ \\
Treatment & $\beta_{0}+\beta_{1}$ & $\beta_{0}+\beta_{1}+\beta_{2}+\beta_{6}$ & $\beta_{0}+\beta_{1}+\beta_{3}+\beta_{7}$ & $\beta_{0}+\beta_{1}+\beta_{4}+\beta_{8}$ & $\beta_{0}+\beta_{1}+\beta_{5}+\beta_{9}$ \\
\hline
\end{tabular}


Table 4 The estimated parameters, their standard errors, the Wald-type $t$-test statistics, and $P$-values resulted from applying the linear mixed-effect model $(I)$ to the current experimental data

\begin{tabular}{|c|c|c|c|c|}
\hline Parameters & Estimate & Standard errors & t-statistics & $P$-values \\
\hline Intercept $\beta_{0}$ & 336.8 & 9.9 & 33.9 & 0.000 \\
\hline Treatment $\beta_{1}$ & 45.5 & 13.9 & 3.3 & 0.001 \\
\hline Zoom rate $100 \% \beta_{2}$ & -1.1 & 0.8 & $\mathrm{I} .4$ & 0.164 \\
\hline Zoom rate $\mathrm{I} 50 \% \beta_{3}$ & -9.6 & 0.7 & -13.9 & 0.000 \\
\hline Zoom rate $200 \% \beta_{4}$ & -11.9 & 0.7 & -17.6 & 0.000 \\
\hline Zoom rate $300 \% \beta_{5}$ & -14.6 & 0.7 & -20.0 & 0.000 \\
\hline Treat: zoom rate $100 \% \beta_{6}$ & 1.7 & I.I & 1.6 & 0.117 \\
\hline Treat: zoom rate $150 \% \beta_{7}$ & 6.6 & 1.0 & 6.9 & 0.000 \\
\hline Treat: zoom rate $200 \% \beta_{8}$ & 6.8 & 0.9 & 7.2 & 0.000 \\
\hline Treat: zoom rate $300 \% \beta_{9}$ & 6.0 & 1.0 & 5.9 & 0.000 \\
\hline$\sigma_{b}$ & 101.6 & & & \\
\hline$\sigma$ & 4.4 & & & \\
\hline
\end{tabular}

indicates that the variances of the measurements are largely contributed by between-myocyte variation. This can also be seen from Figure 2, where the within-myocyte variation is much smaller than the between-myocyte variation.

\section{Measurement accuracy and precision}

Based on the $F$-test statistics for the equality of variances between two groups, the variances of the measurements of myocyte areas between the treated heart and the placebo heart are not significantly different at each zoom rate. Tables 2 and 4 show that the sample means seem to be decreasing when the image zoom rate increases for both the treated heart and the placebo heart. Let us define measurement accuracy as the sample mean, and define measurement precision as the sample variance. ${ }^{4}$ In the following paragraphs we will discuss statistical changes of accuracy and precision as image zoom rate increases.

The plot of the means of the measured areas versus zoom rates is shown in Figure 3. From Figure 3, it is clear that the means of measurements are approximately the same when the zoom rates are $75 \%$ and $100 \%$, whereas the means of measured areas are decreasing as zoom rate increases, and the means of measured areas at zoom rates $150 \%$ and above are significantly smaller than those measured at zoom rate $100 \%$. This decrease is reasonable because the measurement protocol is always trying to trace the inner edges, and the inner edge becomes clearer as the zoom rate increases. Therefore, this might indicate that measurement accuracy would increase as zoom rate increases. These findings are consistent with the findings based on the linear mixed-effect model (1). In addition, we performed paired $t$-tests for comparing the differences of measurements over any two image zoom rates for each type of heart. The results are presented in Tables 5 and 6. Based on Tables 5 and 6, the measurements are not significantly different between zoom rates $75 \%$ and $100 \%$, whereas all the other pairs are significantly different. The results hold for both the treated heart and the placebo heart. We conclude that measurement accuracy is increasing when the image zoom rate increases except between zoom rates at $75 \%$ and $100 \%$ for both the treated heart and the placebo heart.

In addition, we performed an $F$-test for the equality of variances between different zoom rates for each type of heart. All variances were not significantly different across different zoom rates and between the treated heart and the placebo heart. Therefore, it is concluded that with an increasing image zoom rate, measurement precision has no significant improvement.

\section{The optimal zoom rate}

Figure 4 depicts the $t$-statistics corresponding to different image zoom rates. Based on Figure 4, it seems that there is a big jump in the $t$-statistic from zoom rate $100 \%$ to zoom rate $150 \%$, and the $t$-statistics remain at the same level among zoom rates $150 \%, 200 \%$, and $300 \%$.

Because all variances are not statistically different between different zoom rates in both the treated heart and the placebo heart, the pooled variances are not statistically different between any two different zoom rates on the condition of fixed sample sizes.

We examine whether the mean differences between the treated heart and the placebo heart over different zoom rates are significantly different. Denote $\Delta_{75}=\mu_{\mathrm{t} 75}-\mu_{\mathrm{p} 75}, \Delta_{100}=\mu_{\mathrm{t} 100}-\mu_{\mathrm{p} 100}$, $\Delta_{150}=\mu_{\mathrm{t} 150}-\mu_{\mathrm{p} 150}, \Delta_{200}=\mu_{\mathrm{t} 200}-\mu_{\mathrm{p} 200}$, and $\Delta_{300}=\mu_{\mathrm{t} 300}-\mu_{\mathrm{p} 300}$, where $\mu_{\mathrm{t} 75}$ is the mean area of the treated myocyte at zoom rate $75 \%, \mu_{\mathrm{p} 100}$ is the mean area of the placebo myocyte at zoom 


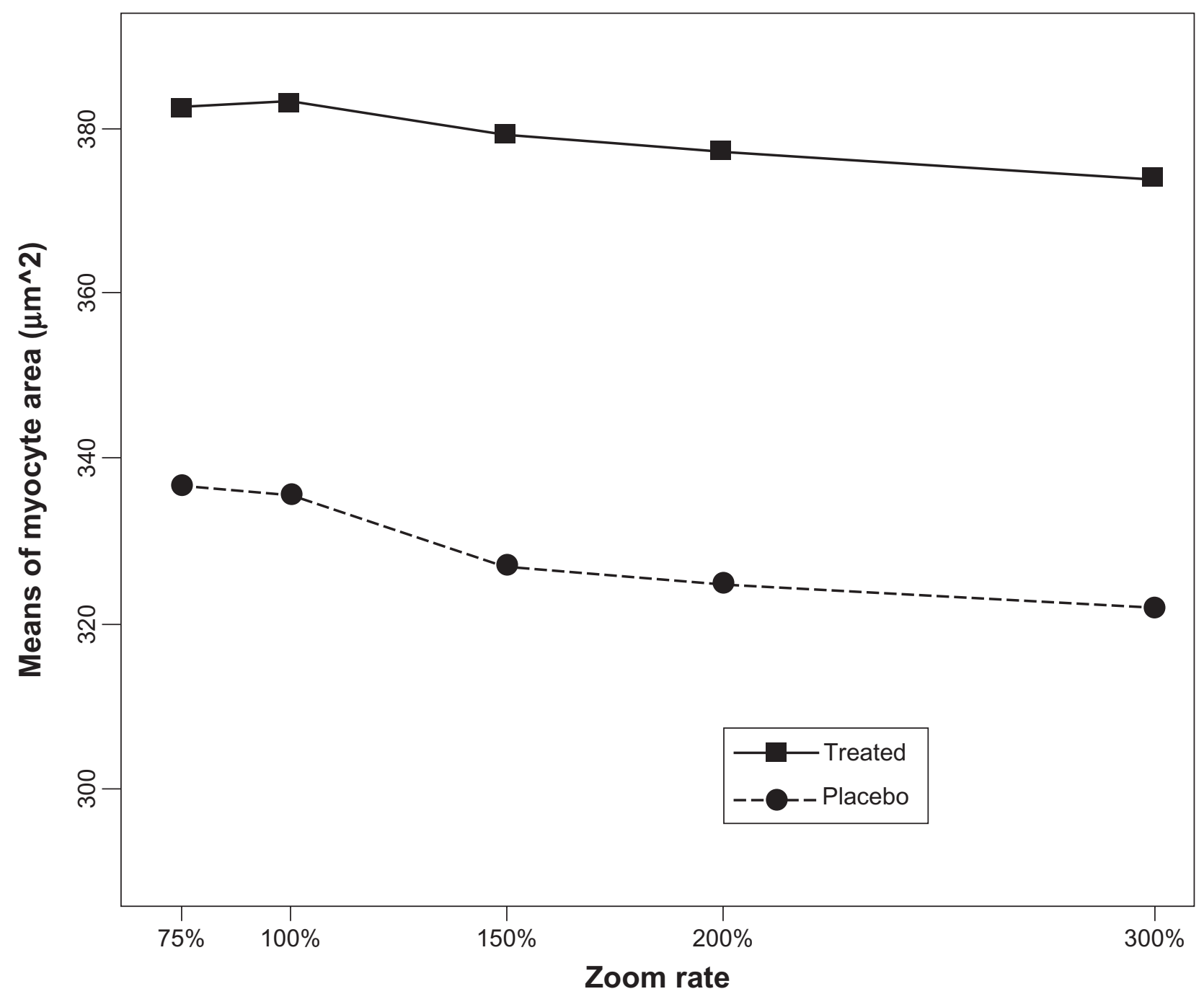

Figure 3 The means of measured myocyte areas over different zoom rates.

Table 5 Pairwise comparisons of means of treated myocytes at different zoom rates

\begin{tabular}{llll}
\hline Comparison & P-value & Comparison & P-value \\
\hline Zoom rate 75\% vs $100 \%$ & 0.923 & Zoom rate 75\% vs 200\% & $<$ le-04 \\
Zoom rate $75 \%$ vs $150 \%$ & 0.00015 & Zoom rate 75\% vs 300\% & $<$ le-04 \\
Zoom rate $100 \%$ vs $150 \%$ & $<$ le-04 & Zoom rate 150\% vs 200\% & $<$ le-04 \\
Zoom rate $100 \%$ vs 200\% & $<$ le-04 & Zoom rate 150\% vs 300\% & $<$ le-04 \\
Zoom rate $100 \%$ vs 300\% & $<$ le-04 & Zoom rate 200\% vs 300\% & $<$ le-04 \\
\hline
\end{tabular}

Table 6 Pairwise comparisons of means for placebo myocytes at different zoom rates

\begin{tabular}{llll}
\hline Comparison & P-value & Comparison & P-value \\
\hline Zoom rate $75 \%$ vs $100 \%$ & 0.616 & Zoom rate 75\% vs 200\% & $<$ e-04 \\
Zoom rate $75 \%$ vs $150 \%$ & $<$ le-04 & Zoom rate 75\% vs 300\% & $<$ e-04 \\
Zoom rate $100 \%$ vs $150 \%$ & $<$ le-04 & Zoom rate $150 \%$ vs 200\% & $<$ e-04 \\
Zoom rate $100 \%$ vs 200\% & $<$ le-04 & Zoom rate 150\% vs 300\% & $<$ e-04 \\
Zoom rate $100 \%$ vs 300\% & $<$ le-04 & Zoom rate 200\% vs 300\% & $<$ le-04 \\
\hline
\end{tabular}




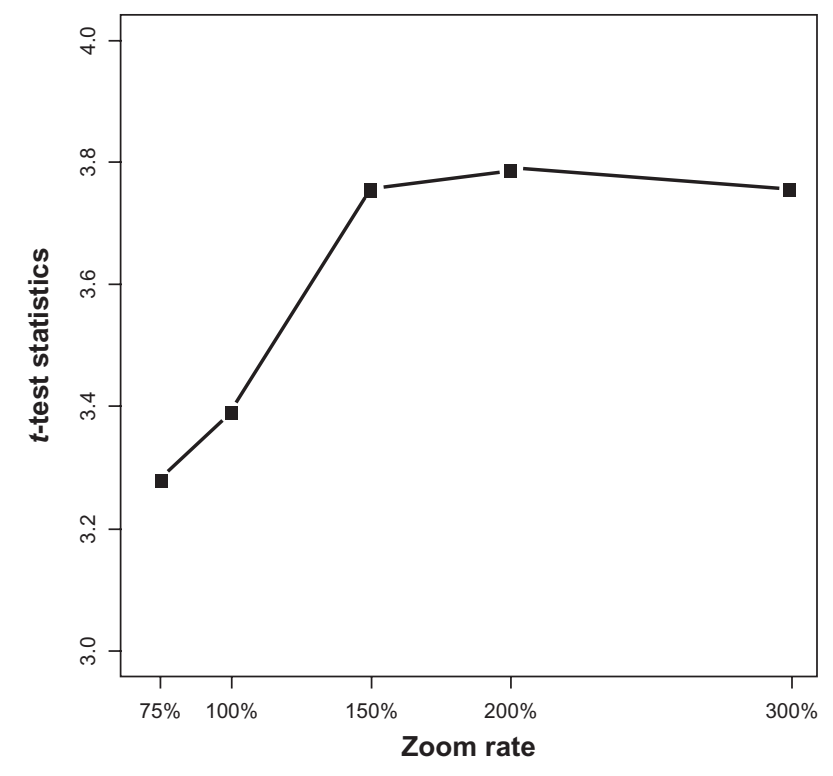

Figure 4 The $t$-test statistic for mean difference between the treated heart and the placebo heart at each zoom rate.

rate $75 \%$, and the other quantities are similarly defined. To test whether the mean differences between the treated heart and the placebo heart over zoom rates $100 \%$ and $150 \%$ are significantly different, the underlying hypotheses are: $\mathrm{H}_{0}$ : $\Delta_{100}=\Delta_{150}$ versus $\mathrm{H}_{1}: \Delta_{100} \neq \Delta_{150}$. The underlying hypotheses can be similarly defined when comparing the other pairs. We applied the $t$-test to test $\mathrm{H}_{0}: \Delta_{100}=\Delta_{150}$ versus $\mathrm{H}_{1}: \Delta_{100} \neq \Delta_{150^{\circ}}$ Because $\Delta_{150}-\Delta_{100}=\left(\mu_{\mathrm{t} 150}-\mu_{\mathrm{p} 150}\right)-\left(\mu_{\mathrm{t} 100}-\mu_{\mathrm{p} 100}\right)=\left(\mu_{\mathrm{t} 150}-\right.$ $\left.\mu_{\mathrm{t} 100}\right)-\left(\mu_{\mathrm{p} 150}-\mu_{\mathrm{p} 100}\right)$, the $F$-test for the equality of variances with the $P$-value of 0.787 indicates that the variances are equal between the measurements for $\left(\mu_{\mathrm{t} 150}-\mu_{\mathrm{t} 100}\right)$ and $\left(\mu_{\mathrm{p} 150}-\mu_{\mathrm{p} 100}\right)$. By using unpaired two-tailed $t$-tests with equal variances, the $P$-value is $3.48 \mathrm{E}-10$, which indicates that there exists a significant difference between $\left(\mu_{\mathrm{t} 150}-\mu_{\mathrm{t} 100}\right)$ and $\left(\mu_{\mathrm{p} 150}-\mu_{\mathrm{p} 100}\right)$. There is a significant difference between $\Delta_{150}$ and $\Delta_{100}$, and apparently $\Delta_{150}>\Delta_{100}$.

Similarly, we compared $\Delta_{200}$ and $\Delta_{150}$. A $P$-value of 0.819 indicates that there is no significant difference between $\left(\mu_{\mathrm{t} 200}-\mu_{\mathrm{p} 150}\right)$ and $\left(\mu_{\mathrm{p} 200}-\mu_{\mathrm{p} 150}\right)$. There is no statistical difference between $\Delta_{200}$ and $\Delta_{150}$, so we cannot reject the null hypothesis $\mathrm{H}_{0}: \Delta_{150}=\Delta_{200}$. In addition, we compared $\Delta_{150}$ and $\Delta_{300}$. A $P$-value of 0.342 indicates that we cannot reject the hypothesis $\mathrm{H}_{0}: \Delta_{150}=\Delta_{300}$. It is concluded that, statistically, $\Delta_{150}>\Delta_{100}, \Delta_{150}>\Delta_{75}, \Delta_{150}=\Delta_{200}$, and $\Delta_{150}=\Delta_{300}$. Because there is no significant difference among the groups for the paired mean differences at different image zoom rates $150 \%, 200 \%$, and $300 \%$, the test statistic remains the same. However, the measurement cost, ie, the time spent to trace the inner edges of these myocytes, is doubled with the zoom rate at $300 \%$ compared with that at $150 \%$. Therefore, we conclude that the image zoom rate of $150 \%$ is the best to achieve the best test power and a low measurement cost.

\section{Conclusion}

As image zoom rate increases, measurement accuracy increases as well. However, measurement precision (variance) has no significant improvement.

Under current measurement conditions, after the image zoom rate reaches the threshold $150 \%$, the mean difference remains a constant statistically. However, the measurement cost would increase dramatically as the image zoom rate increases. We can conclude that the image zoom rate $150 \%$ is optimal in terms of maximizing the test statistic with a low measurement cost.

\section{Discussion}

Because the measurement protocol is defined to trace the inner edge of myocytes, it is reasonable that accuracy will be increased as image zoom rate increases. However, Figure 2 indicates that most of the variations come from the between-myocyte differences for each type of heart, which may explain why the variances between different hearts across different zoom rates are not significantly different.

Achieving maximum accuracy and precision is the general goal in medical quantitative measurement. However, time and effort should also be considered at the current scientific and technical development level. All the measurements should be carried out in an affordable time. The conclusion drawn here may apply to other studies involving measuring area or length with instruments with adjusted zoom rates. The $\mathrm{R}$-codes and the dataset are given in Appendix I and II, respectively.

\section{Disclosure}

The authors report no conflicts of interest in this work.

\section{References}

1. Tang $X$, Rokosh G, Sanganalmath S, et al. Intracoronary administration of cardiac progenitor cells alleviates left ventricular dysfunction in rats with a 30-day-old infarction. Circulation. 2009;12(19):293-305.

2. Pinheiro J, Bates D. Mixed-Effects Models in S and S-Plus. New York, NY: Springer; 2000.

3. Bernard R. Fundamentals of Biostatistics. 6th ed. Boston, MA: Duxbury Resource Center; 2005.

4. Gordis L. Introduction to Epidemiology. 4th ed. Philadelphia, PA: Saunders; 2008 . 


\section{Appendix I: $\mathbf{R}$ codes}

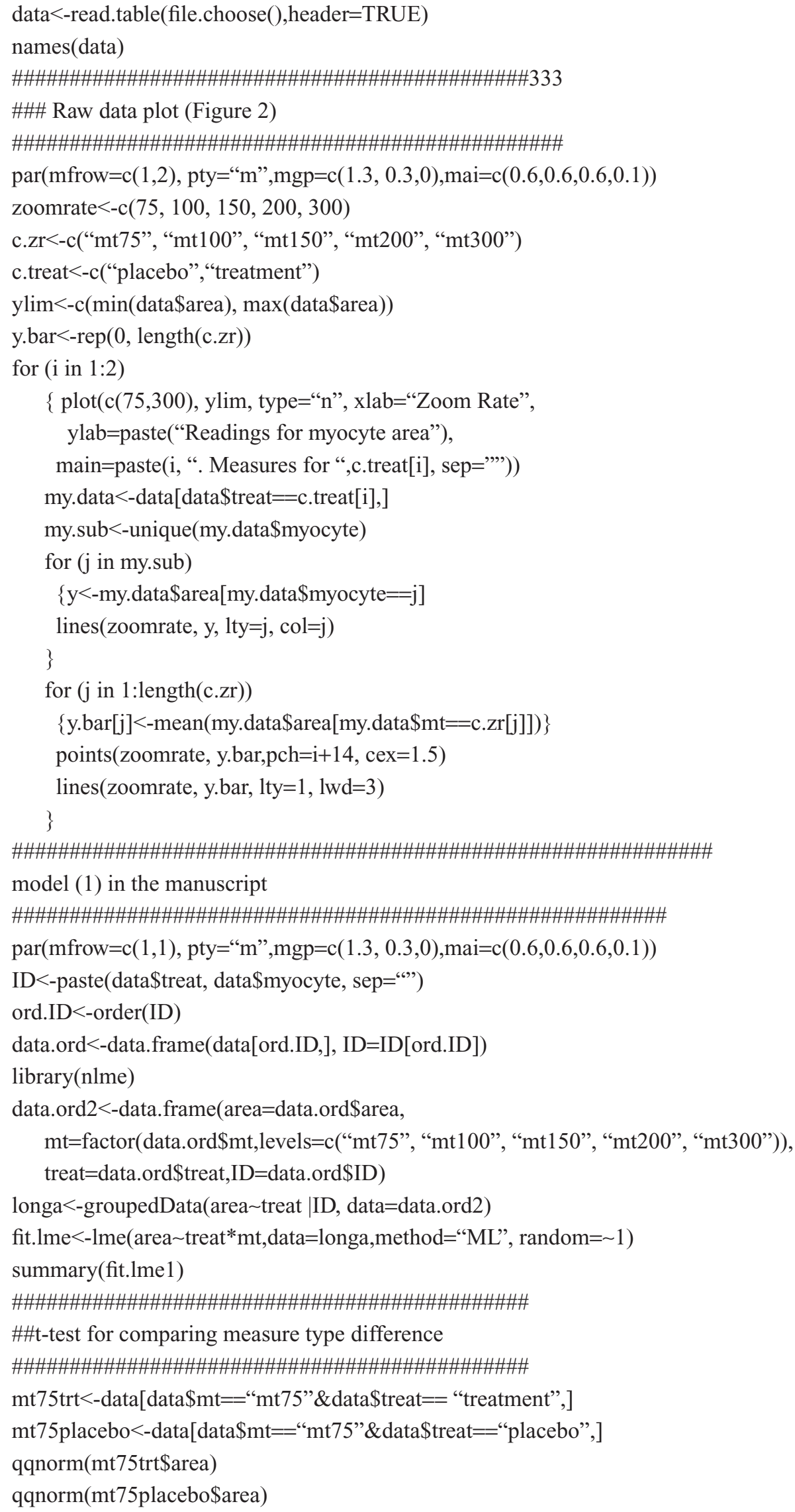




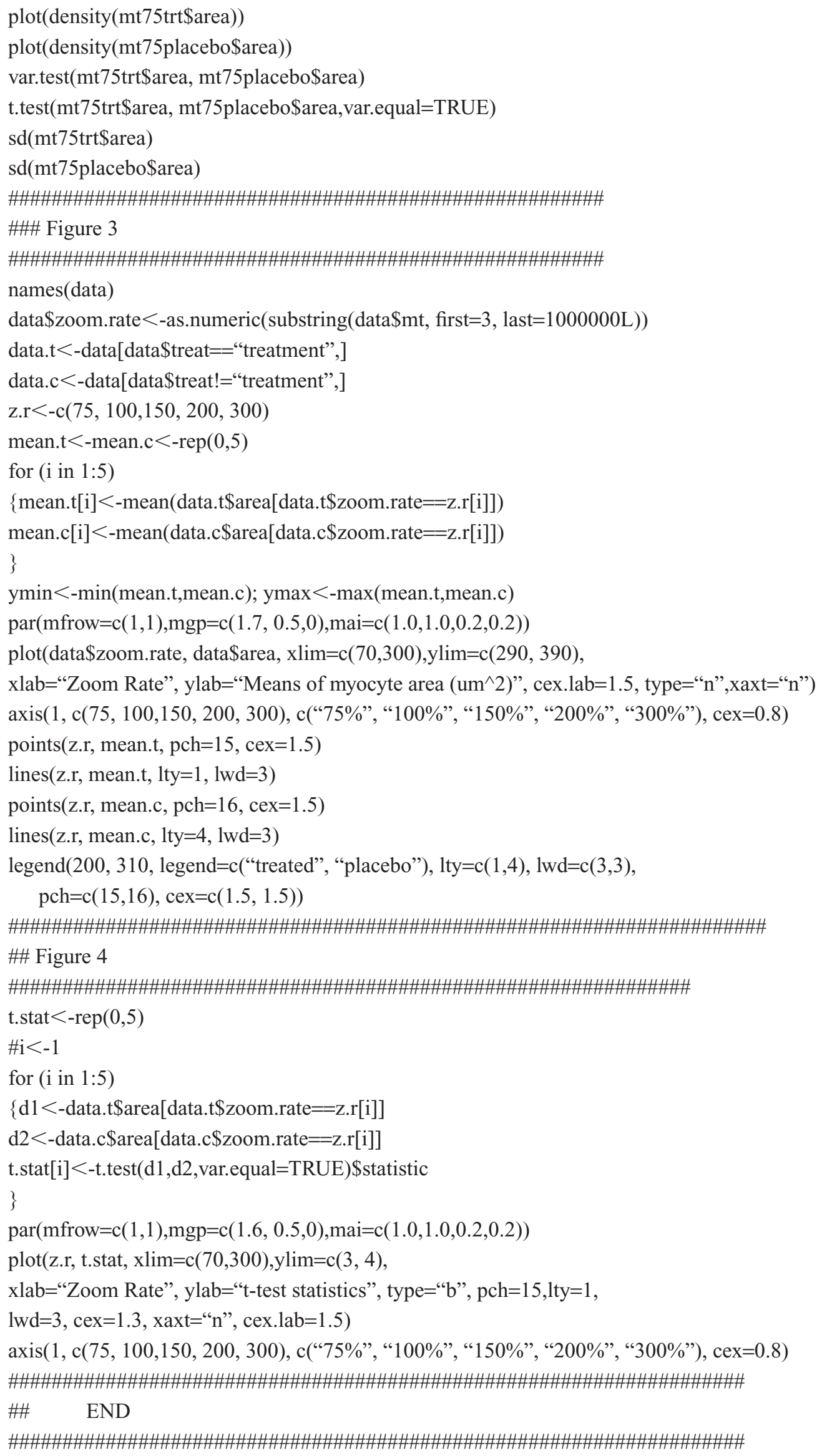




\section{Appendix II: Dataset samples}

\begin{tabular}{|c|c|c|c|}
\hline area & $\mathrm{mt}$ & treat & myocyte \\
\hline 259.509 & $\mathrm{mt} 75$ & treatment & 1 \\
\hline 311.831 & $\mathrm{mt} 75$ & treatment & 2 \\
\hline 255.403 & $\mathrm{mt} 75$ & treatment & 3 \\
\hline 467.642 & $\mathrm{mt} 75$ & treatment & 4 \\
\hline 300.672 & $\mathrm{mt} 75$ & treatment & 5 \\
\hline 323.517 & $\mathrm{mt} 75$ & treatment & 6 \\
\hline 251.297 & $\mathrm{mt} 75$ & treatment & 7 \\
\hline 441.744 & $\mathrm{mt} 75$ & treatment & 8 \\
\hline 341.098 & $\mathrm{mt} 75$ & treatment & 9 \\
\hline 507.437 & $\mathrm{mt} 75$ & treatment & 10 \\
\hline 432.690 & $\mathrm{mt} 75$ & treatment & 11 \\
\hline 288.144 & $\mathrm{mt} 75$ & treatment & 12 \\
\hline 376.366 & $\mathrm{mt} 75$ & treatment & 13 \\
\hline 351.310 & $\mathrm{mt} 75$ & treatment & 14 \\
\hline 465.010 & $\mathrm{mt} 75$ & treatment & 15 \\
\hline 340.783 & $\mathrm{mt} 75$ & treatment & 16 \\
\hline 549.969 & $\mathrm{mt} 75$ & treatment & 17 \\
\hline 280.143 & $\mathrm{mt} 75$ & treatment & 18 \\
\hline 402.054 & $\mathrm{mt} 75$ & treatment & 19 \\
\hline$\ldots$ & $\ldots$ & $\ldots$ & $\ldots$ \\
\hline 314.569 & $\mathrm{mt} 300$ & placebo & 94 \\
\hline 327.307 & $\mathrm{mt} 300$ & placebo & 95 \\
\hline 362.786 & $\mathrm{mt} 300$ & placebo & 96 \\
\hline 286.670 & $\mathrm{mt} 300$ & placebo & 97 \\
\hline 229.189 & $\mathrm{mt} 300$ & placebo & 98 \\
\hline 361.101 & $\mathrm{mt} 300$ & placebo & 99 \\
\hline 535.967 & $\mathrm{mt} 300$ & placebo & 100 \\
\hline 468.484 & $\mathrm{mt} 300$ & placebo & 101 \\
\hline 228.346 & $\mathrm{mt} 300$ & placebo & 102 \\
\hline 514.595 & $\mathrm{mt} 300$ & placebo & 103 \\
\hline 315.306 & $\mathrm{mt} 300$ & placebo & 104 \\
\hline 377.946 & $\mathrm{mt} 300$ & placebo & 105 \\
\hline 379.946 & $\mathrm{mt} 300$ & placebo & 106 \\
\hline
\end{tabular}

Open Access Medical Statistics

\section{Publish your work in this journal}

Open Access Medical Statistics is an international, peer-reviewed, open access journal publishing original research, reports, reviews and commentaries on all areas of medical statistics. The manuscript managepeer-review system. Visit http://www.dovepress.com/testimonials.php to read real quotes from published authors. 